Journal of Mathematics and Informatics

Vol. 20, 2021, 73-89

ISSN: 2349-0632 (P), 2349-0640 (online)

Published 4 May 2021

www.researchmathsci.org

DOI: http://dx.doi.org/10.22457/jmi.v20a08189

Journal of

Mathematics and Informatics

\title{
Sensitivity and Uncertainty Analysis of Variable-Volume \\ Deterministic Model for Endothermic Continuously Stirred Tank Reactor
}

\author{
Jean Pierre Muhirwa ${ }^{1,2, *}$, Isambi Sailon Mbalawata ${ }^{3}$ and Verdiana Grace Masanja ${ }^{1}$ \\ ${ }^{1}$ Nelson Mandela African Institution of Science and Technology Tanzania \\ School of Computational and Communication Science and Engineering \\ Department of Applied Mathematics and Computational Science \\ Arusha-Tanzania, \\ ${ }^{2}$ University of Rwanda-College of Science and Technology, School of Science \\ Department of Mathematics, Kigali-Rwanda, \\ ${ }^{3}$ African Institute for Mathematical Sciences-Secretariat, Research Department, \\ Kigali-Rwanda \\ ${ }^{2}$ E-mail: verdiana.masanja@nm-aist.ac.tz; ${ }^{3}$ E-mail: imbalawata@nexteinstein.org,
}

Corresponding author. Email: muhirwaj@nm-aist.ac.tz,

Received 7 March 2021; accepted 29 April 2021

Abstract. This paper deals with the formulation and the identifiability of the variablevolume deterministic model for the endothermic continuously stirred tank reactor (CSTR). The identifiability of physical parameters of the formulated model is done by using the least squares and the delayed rejection adaptive algorithm version of the Markov chain Monte Carlo (MCMC) method. The least square estimates are used as prior information for the MCMC method. To measure the model output associated with the perturbed model parameters, we use global sensitivity analysis implemented in Latin Hypercube Sampling method. The obtained results from partial rank correlation coefficients show that six parameters are very sensitive and correlated with the model outputs. Finally, we show that the least square and the MCMC numerical results impart the model to be realistic, reliable and worthwhile to describe the dynamics of CSTR processes as physical parameters of the model are well identified and their uncertainties in the model response are analysed and quantified.

Keywords: Variable-volume; Markov chain Monte Carlo; Endothermic; continuously stirred tank reactor

AMS Mathematics Subject Classification (2010): 65C05, 78M31

\section{Introduction}

Continuously stirred tank reactors (CSTRs), as one of the chemical engineering reactors, are recently very useful in industrial production. Chemical engineering reactors are categorised in three main types whereby the first type is batch reactor, the second is semibatch rector and the last is CSTR. The reactors operate differently. For example, the 


\section{J.P. Muhirwa, I.S. Mbalawata and V.G. Masanja}

batch reactor is fed once and the product is removed once whereas for the semi-batch reactor, the reactants are supplied continuously until the product is formed and removed at an instant or vice-versa. On the contrary, CSTR operates in such a way that the reactants are fed continuously and the formed products are also removed continuously. Chemical products that may be produced from those reactors are food supplements, cosmetic products, alcohols, fertilisers, medicines and biogas among others.

CSTRs are very complex and exhibit non-linear operational behaviour which may lead to complex control techniques. For controlling CSTRs, it requires to control the disturbances in inlets and outlets at the same time. The inlets are composed of parameters and variables such as temperature profiles, reactants concentration, reactants density, heating or cooling system temperature, and reactants volume while the outlets are composed of the product concentration, product temperature, product volume, and product density, to name few. Scientists and engineers are interested in fully understanding the dynamics of these tanks through mathematical analysis [1, 2, 3]. Other researchers have carried out uncertainty quantification on CSTR models. For instance, uncertainty quantification of only kinetic parameters on model states of anaerobic digester, which is a kind of CSTR has been studied, analysed and quantified in [4]. Despite the accurate results obtained, it is better to quantify the influence of each and every uncertain parameter on model outputs for the model to be robust.

Many researchers also studied behaviour of the CSTRs with exothermic reactions which release heat energy [5, 6, 7]. However, from the chemical point of view, endothermic reactions which absorb heat energies are equally very important and considered in chemical industries. There are many examples of endothermic reactions that are frequently performed and are abundantly found in nature, in industries, and in our daily real life activities. Few of the known examples include a daily activity of transforming eggs into omelette. This is one of the real examples of endothermic reaction, for which the pan absorbs enough heat energy to cook eggs. Another typical example is plants photosynthesis process, which requires the plants to absorb the sun energy to transform minerals into food. More examples include water evaporation, melting ice cubes, baking breads, and dissolution of salt in water. In modelling the endothermic reaction, some existing researches vary the model parameters around their nominal values instead of sampling them from some distributions, and only consider reacting tank temperature and concentration as state variables or reacting tank temperature, cooling/heating jacket temperature and concentration with the treatment of the volume as a constant $[8,9,10,11]$. It is with these reasons, there is a need to develop an endothermic CSTR model with varying volume and using advanced sampling technique to study and analyse the uncertainty quantification of model parameters. It is important to consider the volume as variable because CSTRs may expand and deform continuously.

This paper aims to consider a four state variables endothermic CSTR deterministic model and sampling and optimizing the CSTR's model parameters by using the least squares and Markov Chain Monte Carlo methods with the motivation of looking at the influence of parameters variability on the system model response.

The reminder of the paper is structured as follows. Section 2 describes the schematic diagrams that show the dynamics of CSTR, the model, assumptions, parameters, constants and variables involved in this research. Section 3 discuses methods and 
Sensitivity and Uncertainty Analysis of Variable-Volume Deterministic Model for Endothermic Continuously Stirred Tank Reactor

materials used. In Section 4, the numerical analysis of the model and discussion of the results are carried out whilst Section 5 gives concluding remarks.

\section{Description of variable-volume deterministic model for endothermic CSTR}

\subsection{CSTR dynamics illustrations}

Figure 1 depicts the dynamics of the endothermic CSTR. On the left hand side, there are physical properties of the raw materials (inlets), and in the center, we have the CSTR under consideration that is covered by the heating jacket which supplies heat energy into CSTR. The collection of products (outlets) with its physical properties from CSTR is on the right hand side. To study the behaviour of the real system as a whole is complicated. That is why a conventional control volume drawn in Figure 2 is involved to represent the Reynold transport theorem (RTT) which governs flows in and out of the system.

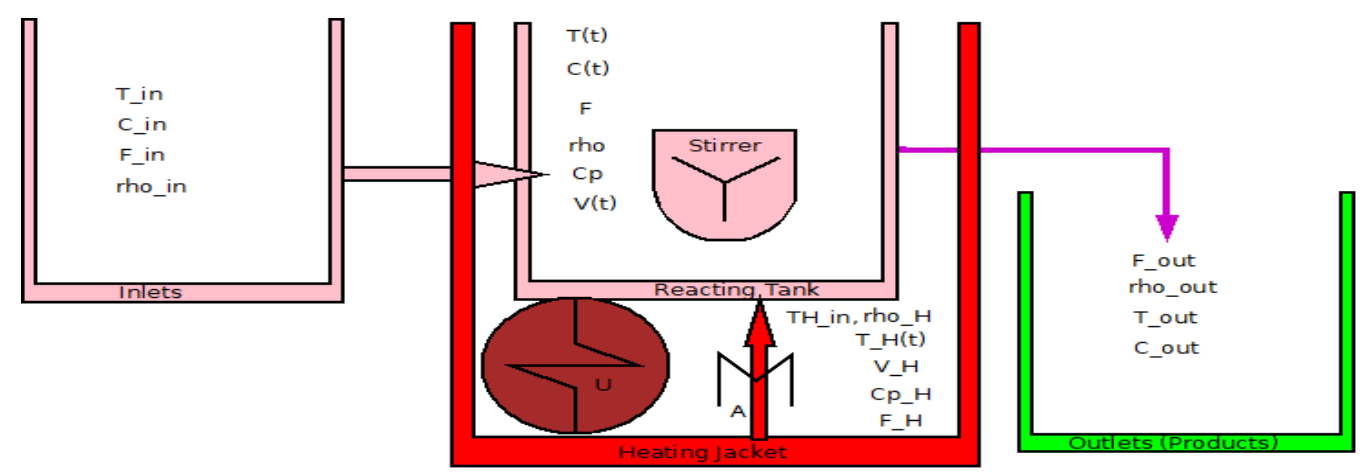

Figure 1: The dynamics of endothermic CSTR process

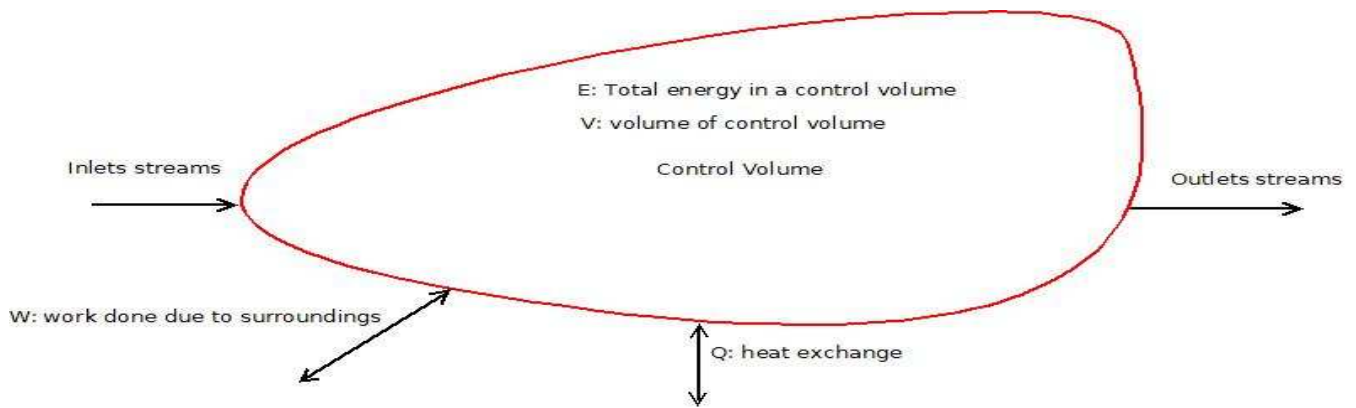

Figure 2: The dynamics of endothermic CSTR using RTT

\subsection{Model assumptions}

Most of mathematical models are built based on assumptions. Therefore, from Figure 2, a variable-volume deterministic model for the endothermic CSTR can be formulated after taking into consideration of the following assumptions:

$A_{1}$ : To avoid spatial gradients of velocity, temperature, concentration and other physical properties of the mixture inside the CSTR, there is a perfect mixing. 


\section{J.P. Muhirwa, I.S. Mbalawata and V.G. Masanja}

$A_{2}$ : Non-viscous fluid and static mixer for which the shaft work produced by the stirring process is negligible.

$A_{3}$ : No pressure drop is taking place in the CSTRs.

$A_{4}$ : Kinetic energy, potential energy and other forms of external energy are infinitesimal small compared to the heat exchange and the heat from the chemical reactions.

$A_{5}$ : Wall temperature is negligible. Only the heat exchange is channelled through the designed area.

$A_{6}$ : The volume is treated as a variable.

$A_{7}$ : Densities $(\rho)$ and specific heat capacities $\left(c_{p}\right)$ are constants.

$A_{8}$ : There is negligible momentum on the system since there is also negligible external stress acting on the system.

Based on the eight assumptions above, the system of Ordinary Differential Equations that governs the dynamics of the deterministic variable-volume model for endothermic CSTR is formulated and it is given by Equation (1);

$$
\left\{\begin{array}{l}
\frac{d V}{d t}=F_{\text {in }}-F_{\text {out }}, \\
\frac{d C}{d t}=\frac{F}{V}\left(C_{\text {in }}-C\right)-k_{0} e^{\frac{-E}{R}\left(\frac{1}{T}-\frac{1}{T_{\text {maan }}}\right)} C, \\
\frac{d T}{d t}=\frac{F}{V}\left(T_{\text {in }}-T\right)+\frac{H^{*} k_{0} e^{\frac{-E}{R}\left(\frac{1}{T}-\frac{1}{T_{\text {mean }}}\right)} C}{\rho c_{p}}+\frac{A U\left(T-T_{H}\right)}{\rho c_{p} V}, \\
\frac{d T_{H}}{d t}=\frac{F_{H}}{V_{H}}\left(T_{H \text { in }}-T_{H}\right)-\frac{A U\left(T-T_{H}\right)}{\rho_{H} c_{p_{H}} V_{H}},
\end{array}\right.
$$

where $V$ denotes the volume of the reactor, $C$ denotes the concentration of the reactant, $T$ denotes the temperature of the mixture inside the tank, $T_{H}$ denotes the temperature inside the covering heating jacket, $F_{\text {in }}$ denotes the inlet volumetric flow rate, $F_{\text {out }}$ represents the outlet volumetric flow rate, $F$ is the overall volumetric flow rate of the mixture, $C_{i n}$ is the inlet concentration, $k_{0}$ is the pre-Arrhenius frequency factor, $E$ is the activation energy, $R$ is the gas law constant, $T_{\text {mean }}$ is the reference temperature, $T_{i n}$ is the inlet temperature, $H^{*}$ is the reaction enthalpy, $\rho$ is the mixture density, $c_{p}$ is the heat capacity of the reactor, $A$ is the cross-sectional area between the reactor and the heating jacket to allow the heat energy flow, $U$ is the heat transfer coefficient, $F_{H}$ is the volumetric flow rate of the heater, $V_{H}$ is the volume of the heating jacket, $T_{H i n}$ is the heating temperature inlet, $\rho_{H}$ is the density of the heater, and $c_{p_{H}}$ is the heat capacity of the heating jacket. 
Sensitivity and Uncertainty Analysis of Variable-Volume Deterministic Model for Endothermic Continuously Stirred Tank Reactor

\section{Methods and materials}

\subsection{Least squares method}

The least squares method is among the classical optimization methods that aim to minimize the sum of squares of the model's residuals [12]. The residual is defined as the deviation of the predicted model values from the observed model values or simply the model bias. Mathematically, given the model function $y=f(x(t), \beta)+r$, then $r$ is the residual while $f(x(t), \beta)$ is the numerical solutions of the model (observed values), $x(t)$ is the vector of model dependent variables, $\beta$ is the vector of model parameters, and $\mathrm{y}$ is the predictive dependent model. Thus, from Equation 1, we have

$$
x(t)=\left[V(t), C(t), T(t), T_{H}(t)\right]
$$

and $\beta=\left[F_{\text {out }}, F, k_{0}, E, T_{\text {mean }}, H^{*}, \rho, c_{p}, U, A, F_{H}, V_{H}, \rho_{H}, c_{p H}\right]^{\prime}$. In order to use the least squares method, one needs to have experimental data but due to technology advancement and due to the development of computers, simulations become a very useful approach to experimentally describe the physical systems. In addition, various applied mathematics and engineering problems are enough complicated to be solved analytically and to be experimentally determined due to non-linearity behaviors as mentioned in [13]. For instance, in this paper, as we have the degree of freedom which is fourteen, we need to minimize the sum of squares of residuals by solving fourteen nonlinear systems of equations simultaneously. Analytically, this task seems to be complex. As a result, simulations simplify the task and minimize the cost and other unnecessary risks that may occur during statistical experimental studies. To obtain the statistical experimental data, we introduce the noise of standard deviation 0.05 to hundred numerical solutions (100 datasets) of the model Equation (1) which is equivalent to 100x4 statistical experimental datasets. The obtained corrupted data points are then the statistical experimental measurements of the variable-volume deterministic model for the endothermic CSTR.

\subsection{Markov chain Monte Carlo method}

Markov chain Monte Carlo (MCMC) method is among the recent advanced sampling techniques developed to tackle the estimation of parameters of complex systems such as biological, chemical and engineering systems. As an example, the method has been used to study the dark energy model with gravitational lens in [14]. It has been also used as an approximation method for branching process of signal processing in [15]. The ingredients of the MCMC method comprise of a distribution called the proposal distribution, initial covariance matrix, and prior information about model parameters. In this paper, initial parameter values are optimized by using least squares method and treated as the prior information about model parameters for the MCMC method. The suitable proposal distribution used for this case is chosen to be Gaussian and the turned initial covariance matrix is $\Sigma_{0}=\frac{0.00001 \times I_{d \times d}}{\sqrt{d}}$, where $d$ represents the number of parameters to be identified, and $I_{d \times d}$ is the $d \times d$ identity square matrix. We generate 500,000 samples for each one of the 14 parameters. Finally, statistical inference and graphical analysis, 


\section{J.P. Muhirwa, I.S. Mbalawata and V.G. Masanja}

which are trace, scatter, autocorrelation, histograms and marginal density distributions are performed to study the convergence and the identifiability of the model parameters. The delayed-rejection adaptive metropolis (DRAM) as one of the types of the MCMC algorithms which combines two concepts of delayed-rejection (DR) and adaptive metropolis (AM) is used to efficiently and accurately enhance the sampling of posterior distributions, especially for complex and non-linear models which sometimes have a slow startup sampling. This method overcomes the MCMC routines of the burn-in and thinning of some posterior samples during sampling period. The DRAM algorithm used in this paper, is also found in $[16,17,18,19]$, and it is presented as follow:

Step 1: Initialization of the algorithm: Get the initial $\beta_{0}$ from the initial proposal distribution $p_{0}(\beta)$ and set the initial non-adaptive period $N_{0}$ and the initial covariance matrix $\Sigma_{0}$.

Step 2: Implementation of the algorithm: For $j=1,2 \ldots$ do the following:

(i) Sample a current point of $\beta$ from the current proposal distribution $q\left(\beta / \beta_{j-1}\right)$,

(ii) Compute the acceptance probability $\alpha$ with probability $p$ as

$$
\alpha\left(\beta_{j-1}, \beta\right)=\min \left\{1, \frac{p\left(\beta / X_{1}, X_{2}, \ldots, X_{n}\right) q\left(\beta_{j-1} / \beta\right)}{p\left(\beta_{j-1} / X_{1}, X_{2}, \ldots, X_{n}\right) q\left(\beta / \beta_{j-1}\right)}\right\},
$$

Accept $\beta_{j}=\beta$ if $v<\alpha\left(\beta_{j-1}, \beta\right)$, where $v \square U(0,1)$,

Otherwise reject the drawn sample point and keep $\beta_{j-1}=\beta$.

(iii) After each iteration, update the covariance matrix with the formula $\Sigma_{j}=\operatorname{Cov}\left(\beta_{0}, \beta_{1}, \ldots, \beta_{j}\right)+\xi I_{p \times p}$, where $\xi$ is a small non-negative number that avoids the covariance matrix to be singular,

(iv) $\quad j \leftarrow j+1$.

\section{Numerical analysis of the model}

The numerical solutions for the deterministic variable-volume endothermic CSTR model (1) are performed by using the fourth order Runge-Kutta method which is an ode45solver software package available in MatlabR2016b.

\subsection{Numerical simulations}

The simulation of model (1) requires parameters and initial values. Table 1 shows parameters and initial values used to obtain the numerical solutions of the model, and the graphical presentation of the state variables' solutions are shown in Figure 3. Also, the samples are generated and analysed graphically using trace, scatter, autocorrelation, histogram and marginal density distribution plots. 
Sensitivity and Uncertainty Analysis of Variable-Volume Deterministic Model for Endothermic Continuously Stirred Tank Reactor

Table 1: parameter values, constants and variables.

\begin{tabular}{|c|c|c|c|}
\hline Parameter Symbol (unit) & $\begin{array}{l}\text { Physical } \\
\text { Meaning }\end{array}$ & $\begin{array}{l}\text { Literature } \\
\text { Value }\end{array}$ & Reference \\
\hline$C_{i n}\left(\frac{k m o l}{\min m^{3}}\right)$ & $\begin{array}{l}\text { Feeding } \\
\text { Concentration }\end{array}$ & 316.8 & {$[20]$} \\
\hline$C_{0}\left(\frac{\mathrm{kmol}}{\min \mathrm{m}^{3}}\right)$ & $\begin{array}{l}\text { Initial } \\
\text { Concentration }\end{array}$ & 316.8 & [20] \\
\hline$C\left(\frac{\mathrm{kmol}}{\min m^{3}}\right)$ & $\begin{array}{l}\text { Mixture } \\
\text { Concentration }\end{array}$ & State Variable & Simulated \\
\hline$T_{i n}(K)$ & $\begin{array}{l}\text { Feeding } \\
\text { Temperature }\end{array}$ & 298.35 & [20] \\
\hline$T_{0}(K)$ & $\begin{array}{l}\text { Initial } \\
\text { Temperature }\end{array}$ & 298.35 & [20] \\
\hline$T(K)$ & $\begin{array}{l}\text { Mixture } \\
\text { Temperature }\end{array}$ & State Variable & Simulated \\
\hline$H^{*}\left(\frac{k c a l}{k m o l}\right)$ & $\begin{array}{l}\text { Reaction } \\
\text { Enthalpy }\end{array}$ & $1004.3 \times 10^{3}$ & [20] \\
\hline$T_{H_{0}}(K)$ & $\begin{array}{l}\text { Initial Heating } \\
\text { Temperature }\end{array}$ & 288.15 & [20] \\
\hline$T_{\text {Hin }}(K)$ & $\begin{array}{l}\text { Feeding } \\
\text { Heating } \\
\text { Temperature }\end{array}$ & 293 & [21] \\
\hline$T_{H}(K)$ & $\begin{array}{l}\text { Heating } \\
\text { Temperature }\end{array}$ & State Variable & Simulated \\
\hline$R\left(\frac{k J}{k m o l}\right)$ & $\begin{array}{ll}\text { Gas } & \text { Law } \\
\text { Constant } & \end{array}$ & 8.314 & [20] \\
\hline$V_{0}\left(m^{3}\right)$ & Initial Volume & 100 & [22] \\
\hline$V\left(m^{3}\right)$ & $\begin{array}{l}\text { Reactor } \\
\text { Volume }\end{array}$ & State Variable & Simulated \\
\hline$k_{0}\left(\frac{1}{\min }\right)$ & $\begin{array}{l}\text { Pre-Arrhenius } \\
\text { Frequency }\end{array}$ & 0.9 & {$[20]$} \\
\hline$E\left(\frac{k J}{k m o l}\right)$ & $\begin{array}{l}\text { Activation } \\
\text { Energy }\end{array}$ & 0.5 & [20] \\
\hline$T_{\text {mean }}(K)$ & $\begin{array}{l}\text { Reference } \\
\text { Temperature }\end{array}$ & 298.15 & [20] \\
\hline$F=F_{i n}\left(\frac{m^{3}}{\min }\right)$ & $\begin{array}{l}\text { Volumetric } \\
\text { Flow Rate }\end{array}$ & $130 \times 10^{-2}$ & [20] \\
\hline$\rho\left(\frac{k g}{m^{3}}\right)$ & $\begin{array}{l}\text { Density of the } \\
\text { Mixture }\end{array}$ & 1000 & [22] \\
\hline
\end{tabular}


J.P. Muhirwa, I.S. Mbalawata and V.G. Masanja

\begin{tabular}{|c|c|c|c|}
\hline$c_{p}\left(\frac{k c a l}{k g K}\right)$ & $\begin{array}{l}\text { Specific Heat } \\
\text { Capacity }\end{array}$ & 4186 & [20] \\
\hline$U\left(\frac{k J}{\min m^{2} K}\right)$ & $\begin{array}{l}\text { Heat Transfer } \\
\text { Coefficient }\end{array}$ & 100000 & Assumed \\
\hline$A\left(m^{2}\right)$ & $\begin{array}{l}\text { Cross-sectional } \\
\text { Area }\end{array}$ & 0.015 & [20] \\
\hline$F_{H}\left(\frac{m^{3}}{\min }\right)$ & $\begin{array}{l}\text { Volumetric } \\
\text { Flow of the } \\
\text { Heater }\end{array}$ & $46.5 \times 10^{-6}$ & [20] \\
\hline$V_{H}\left(m^{3}\right)$ & $\begin{array}{l}\text { Heating } \\
\text { Reactor } \\
\text { Volume }\end{array}$ & $50 \times 10^{-6}$ & [20] \\
\hline$\rho_{H}\left(\frac{k g}{m^{3}}\right)$ & Heater Density & 1000 & [22] \\
\hline$c_{p H}\left(\frac{k c a l}{k g K}\right)$ & $\begin{array}{l}\text { Heater Specific } \\
\text { Heat }\end{array}$ & 4186 & [20] \\
\hline$F_{\text {out }}\left(\frac{m^{3}}{\min }\right)$ & $\begin{array}{l}\text { Outlet } \\
\text { Volumetric } \\
\text { Flow rate }\end{array}$ & $130 \times 10^{-6}$ & [20] \\
\hline
\end{tabular}
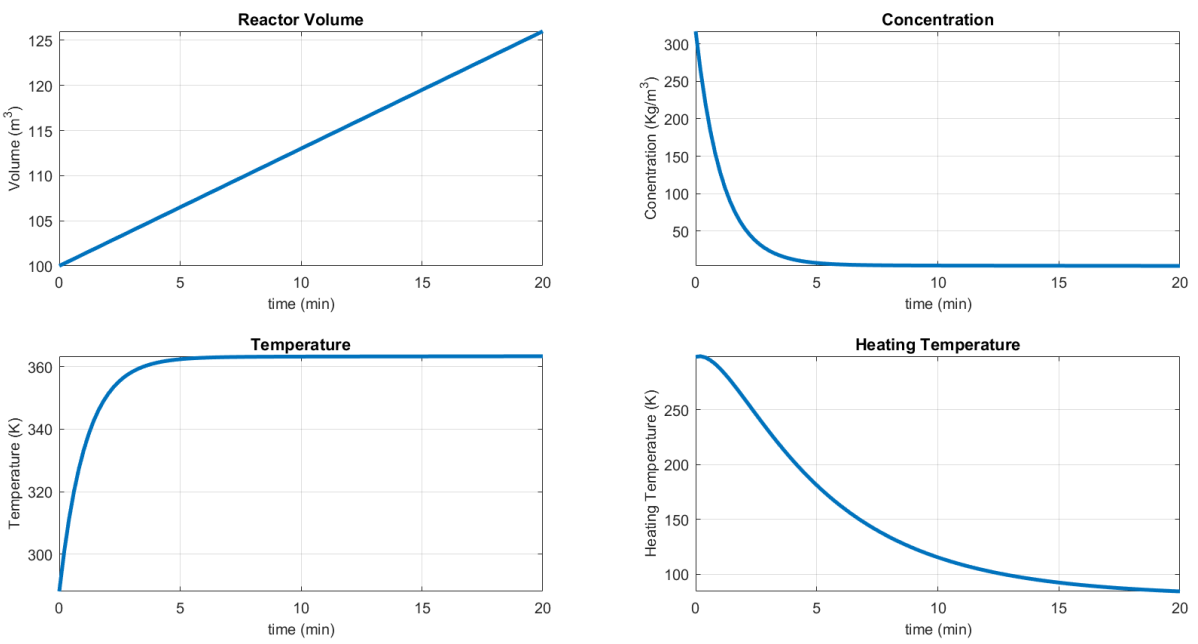

Figure 3: Numerical results of the model

From Figure 3, we can see that the concentration of the reactants is decreasing inside the tank from 316.8 and approaching zero. When the reactants are fed continuously into the tank they are consumed and this is an indication of having a complete conversion of reactant's concentration into product's concentration. The temperature of the system is observed to increase exponentially from $298.35^{\circ} \mathrm{K}$ to $360^{\circ} \mathrm{K}$, however there is a covering heating process to boost the reactor's temperature. Consequently, the temperature of the 
Sensitivity and Uncertainty Analysis of Variable-Volume Deterministic Model for Endothermic Continuously Stirred Tank Reactor

heating tank decreases slightly from $288.15^{\circ} \mathrm{K}$ to almost zero after 5 minutes. From subplot 1 of Figure 3, we can further observe that the volume of the reacting tank increases with time as the feeding rates become more and greater than the removal rates. This has a significance in designing the reacting tank which may deform continuously to increase the production conversion rate from $100 \mathrm{~m}^{3}$ to approximately $126 \mathrm{~m}^{3}$.

\subsection{Least squares results}

To apply the least squares, we initialize the model parameter values as $\beta_{0}=\left[F_{\text {out }}, F, k_{0}, E, T_{\text {mean }}, H^{*}, \rho, c_{p}, U, A, F_{H}, V_{H}, \rho_{H}, c_{p H}\right]^{\prime}$

$=\left[130 \times 10^{-6}, 130 \times 10^{-2}, 0.9,0.5,298.15,1004.3 \times 10^{3}, 1000\right.$,

$\left.4186,100000,0.015,46.5 \times 10^{-6}, 50 \times 10^{-6}, 1000,4186\right]^{\prime}$.

We also initialize the state variables of the system as $x_{0}=[100,316.8,298.35,288.15] '$.

From Figure 4, we can observe that the distance between the predictive solutions and the exact model solutions seems to be minimized. Hence the system model is fitting the measurements very well, simply because red and blue colors are nearly coincident.
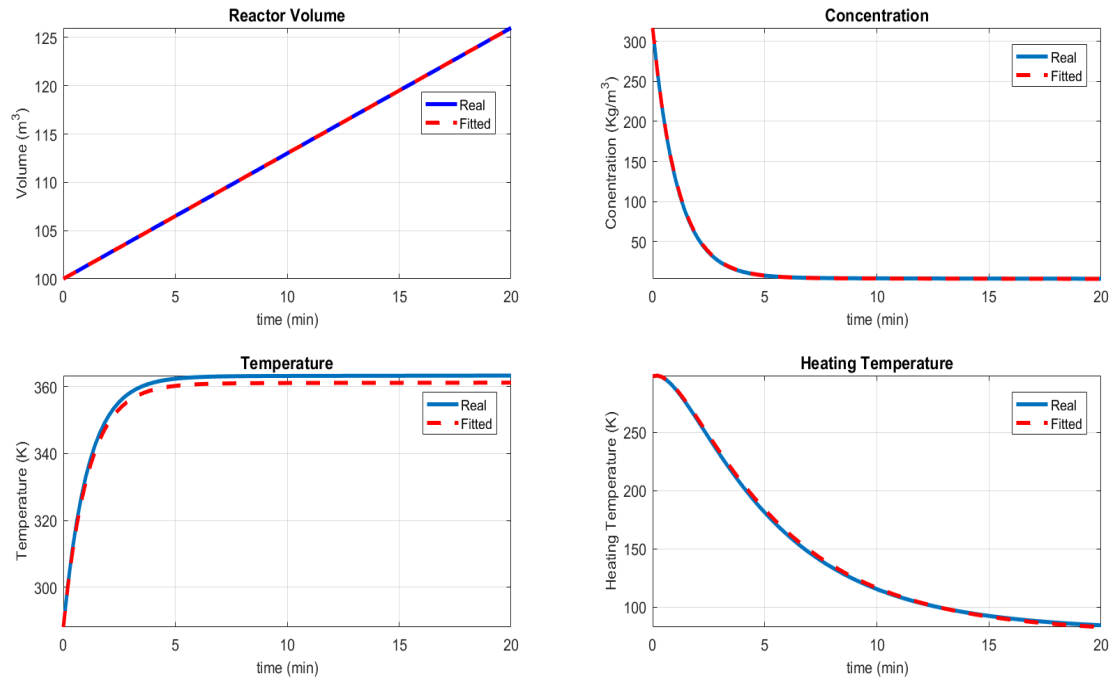

Figure 4: The fitted model. The real data are experimental data after introducing noise.

\subsection{MCMC results and uncertainty analysis}

The identifiability of the model parameters in MCMC is mainly based on the convergence diagnostic tests and the uncertainty analysis of posterior distributions. Various diagnostic tests for MCMC convergence and uncertainty analysis include observatory tests, statistical tests, and graphical tests [23, 24, 25, 26, 27, 28, 29, 30, 31]. In this paper, the MCMC graphical diagnostic tests used are trace (time-series), scatter (pairs), histograms of the posterior distributions and autocorrelation plots. The statistical tests are performed to check, examine and quantify some statistical quantities of the 


\section{J.P. Muhirwa, I.S. Mbalawata and V.G. Masanja}

posteriors distributions. The statistical tests used are posterior means, medians, standard deviations, MCMC errors, tau, geweke, kurtosis and skewness.

\subsubsection{Trace plots}

The generated time series for parameters is plotted with the target of diagnosing whether the posterior parameter distributions are stationary. If the chain gets stuck somewhere during sampling period, then the chain does not move straightforward from one side to another. So, the MCMC algorithm produces a poor mixing. The poor mixing indicates that the parameters are not identifiable. Therefore, further task of changing the ingredients of the algorithm should be done to efficiently and effectively identify the parameters.
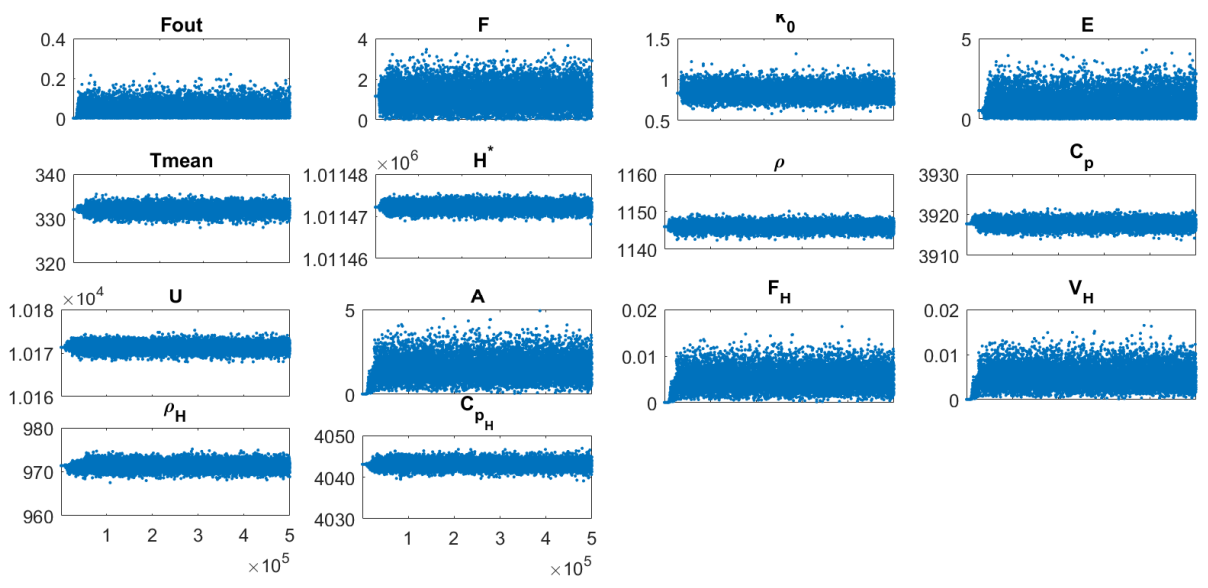

Figure 5: Trace plots of sampled posteriors

Trace plots that are shown in Figure 5 indicate that there are no high trends in sampled parameters since the chain is stationary and moves from one side to another. We may explore that DRAM does not stuck in any place during sampling, which represents the good mixing of the chain.

\subsubsection{Scatter plots}

The scatter plots check the correlation index between pairs of samples. High correlation index among many pairs of samples can lead to poor identifiability of the model parameters. As a consequence, the model cannot be reliably applicable. Low correlation index among pairs is preferably to be observed for a good mixing. 
Sensitivity and Uncertainty Analysis of Variable-Volume Deterministic Model for Endothermic Continuously Stirred Tank Reactor

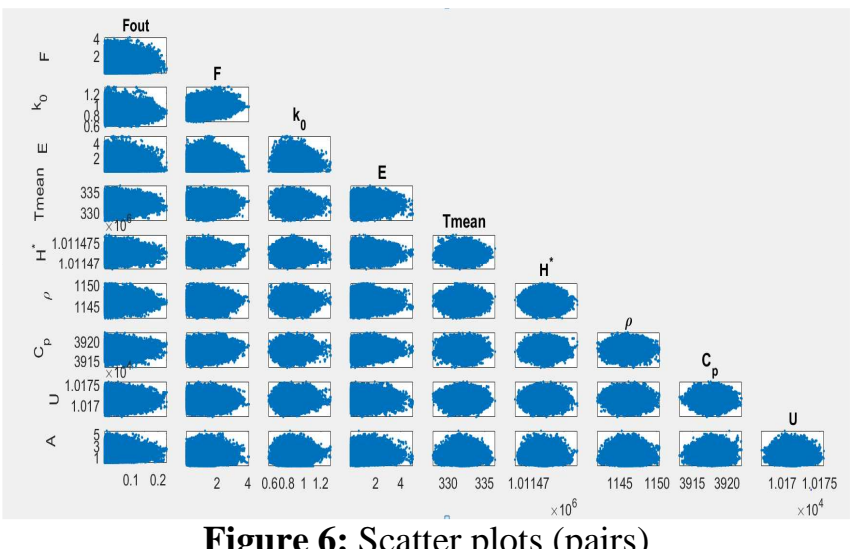

Figure 6: Scatter plots (pairs)

As there are fourteen parameters to be identified from the data points, then there are as many as combinations as possible for each of the chosen pairs from the fourteen parameters and this is equivalent to ninety one scatter plots. However, we only present the scatter plots of the first ten parameter samples which is equivalent to forty five scatter plots. Figure 6 shows that none of the parameters are strongly correlated with each other. If one observes strong correlation among sampled parameters, then it is the indication that the algorithm mixes badly.

\subsubsection{Histograms for posteriors}

The histograms for sampled posterior parameters for a converging Markov chain must fairly follow the normal distribution curve. It is therefore a good practice to plot the histograms for all sampled parameters to make sure that all of them have bell shapes.
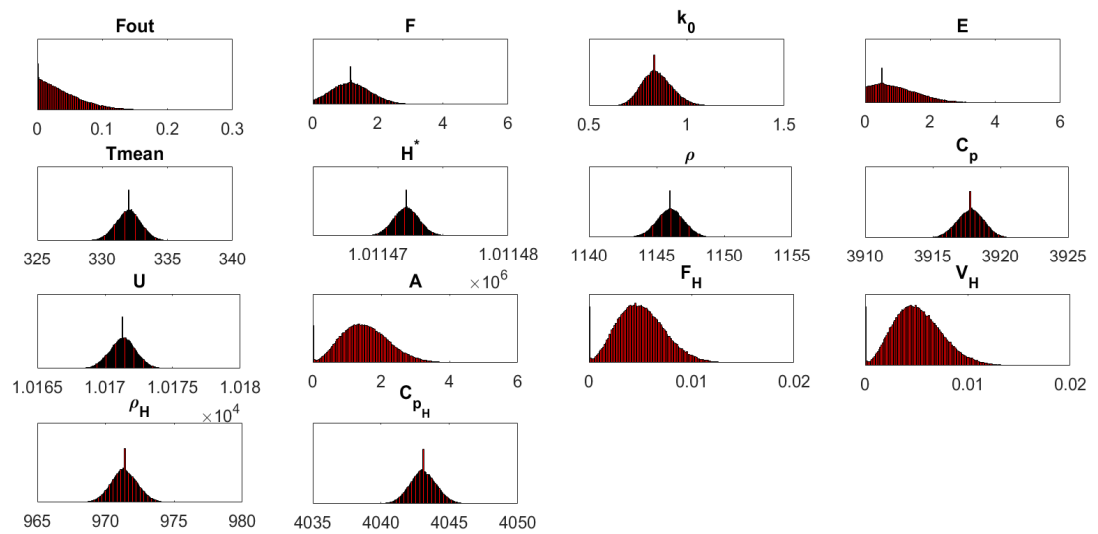

Figure 7: Histograms for the posterior samples distribution

So, from Figure 7, we see that almost all sampled parameters agree with the normal distributions, except for the parameters $F_{\text {out }}$ and $E$ which are slightly skewed to the right side. As a result, the DRAM method identified well the parameters of the model (1). 


\section{J.P. Muhirwa, I.S. Mbalawata and V.G. Masanja}

\subsubsection{Autocorrelation plots}

The autocorrelation plots show how independent is the sampling process. If the coefficients of the autocorrelation functions (x-axis) for some of the posteriors do not decay toward zero as the number of lags (y-axis) increases, then it is the sign of having parameters dependence in sampling which causes the MCMC method not to converge to the target distribution. Thus, a decay of autocorrelation functions coefficients provides the accuracy and the certainty of obtaining a converging posterior distribution of parameters.

From Figure 8, we can see that all coefficients of autocorrelation functions are exponentially decaying as the number of lags increases. Therefore, the consecutive parameters are independently sampled during the run-time of the DRAM algorithm, and this determines the convergence of the method.
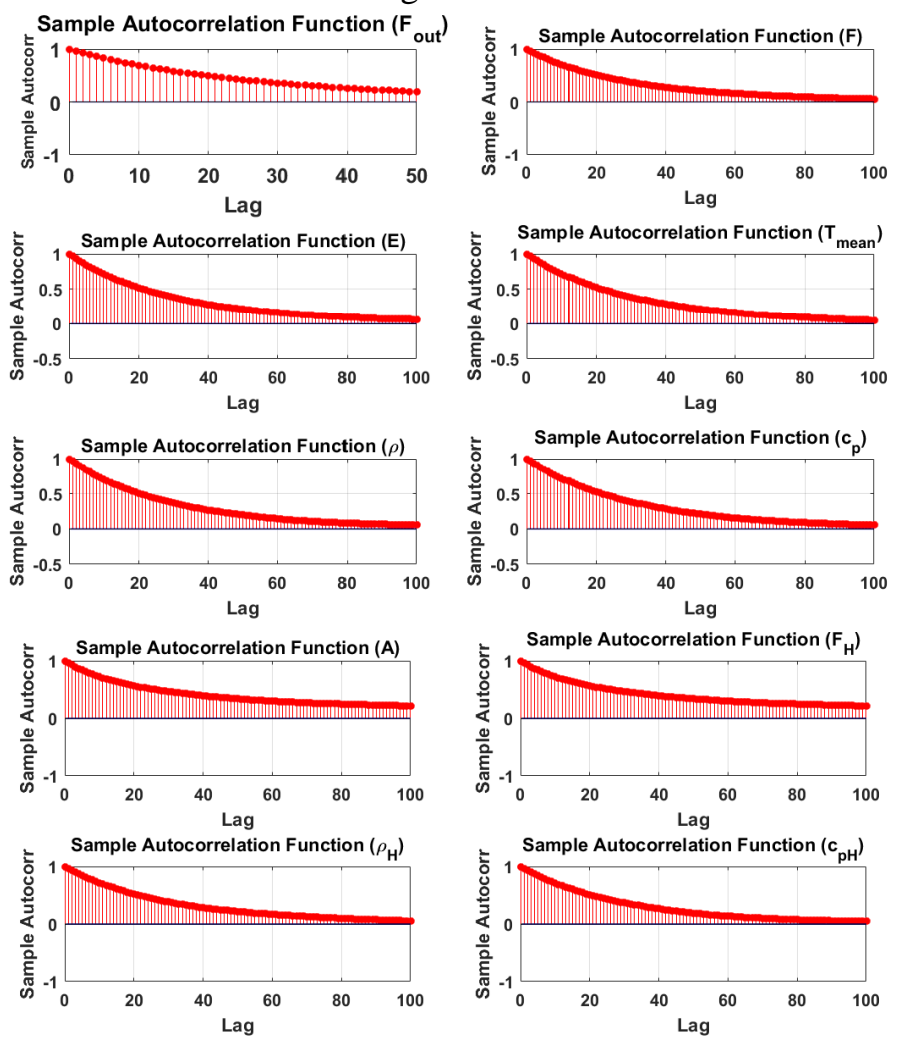
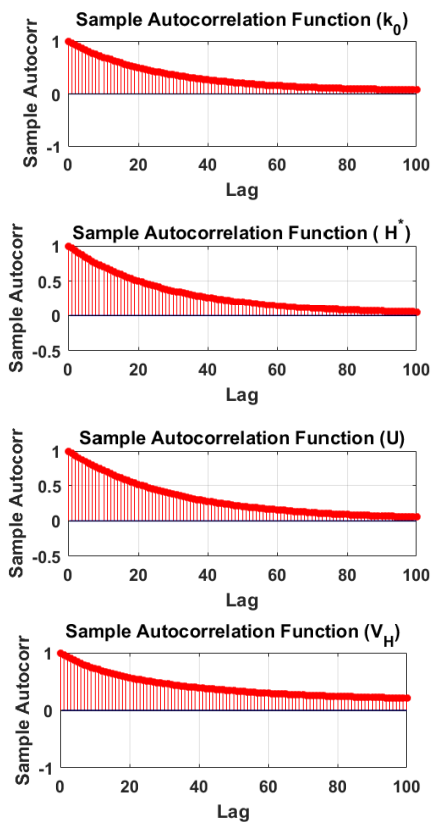

Figure 8: Autocorrelation functions for all 14 identified parameters

The results in Table 2 show that all posterior means are within their credible intervals. The MCMC method converges as the MCMC deviations (MCerr) are minimized. Some curves for posterior means have shown skewness and deviate from normal curves for example $F_{\text {out }}$. This has been justified by the kurtosis and the skewness values of $F_{\text {out }}$ that are far from 3 and 0 respectively, as it can be expected from Gaussian distribution. This indicates that most of all parameters follow Gaussian distribution. The geweke numbers for posterior distributions also check the convergence of the MCMC method and by default assuming that the posterior means for two windows which are the 
Sensitivity and Uncertainty Analysis of Variable-Volume Deterministic Model for Endothermic Continuously Stirred Tank Reactor

start of sampling (10\%) and the end of sampling (50\%) are identical. When samples are drawn from stationary distribution then geweke diagnostic statistical test approaches standard normal distribution and its values are expected to be at most near unity for converging chain. While tau values indicate the autocorrelation times and so the decrease in tau values implies the better mixing in sampling a certain posterior parameter distribution. Based on that, the geweke and tau values obtained in Table 2 show that there were better mixing and independence in sampling posteriors from stationary distribution as geweke values for all parameters are at most nearly unity and tau values are small.

Table 2: Estimated parameters and their statistical inferences

\begin{tabular}{|c|c|c|c|c|c|c|c|c|c|}
\hline Param & \begin{tabular}{|l} 
LSQ \\
estimates
\end{tabular} & $\begin{array}{l}\text { Posterior } \\
\text { mean }\end{array}$ & $\begin{array}{l}\text { Posterior } \\
\text { Standard } \\
\text { deviation }\end{array}$ & $\begin{array}{l}\text { Credible } \\
\text { interval }\end{array}$ & MCerr & tau & geweke & Skewness & Kurtosis \\
\hline$F_{\text {out }}$ & $\begin{array}{l}127.429 \\
\times 10^{-6} \\
\end{array}$ & 0.040469 & 0.033356 & \begin{tabular}{|l|}
{$[0.040377$,} \\
$0.0405619]$
\end{tabular} & 0.000911 & 72.252 & 0.75014 & 1.15 & 4.3 \\
\hline$F$ & $\begin{array}{l}122.894 \\
\times 10^{-2} \\
\end{array}$ & 1.1983 & 0.58335 & $\begin{array}{l}{[1.196687,} \\
1.199921]\end{array}$ & 0.008309 & 59.379 & 0.99404 & 0.3499 & 2.9 \\
\hline$k_{0}$ & 0.8881 & 0.84984 & 0.079068 & \begin{tabular}{|l}
{$[0.849618$,} \\
$0.850056]$
\end{tabular} & 0.000664 & 57.624 & 0.99733 & 0.398 & 3.4 \\
\hline$E$ & 0.5097146 & 0.99429 & 0.69412 & $\begin{array}{l}{[0.992368,} \\
0.996216]\end{array}$ & 0.022557 & 71.166 & 0.782 & 0.849 & 3.4 \\
\hline$T_{\text {mean }}$ & 295.29 & 332 & 0.97761 & $\begin{array}{l}{[331.9976,} \\
332.00305]\end{array}$ & 0.011375 & 66.184 & 0.99982 & -0.0325 & 3.1 \\
\hline$H^{*}$ & 997444.32 & 1011500 & 0.96855 & 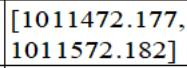 & 0.018189 & 60.161 & 1 & 0.0119 & 3.05 \\
\hline$\rho$ & 1094.291 & 1146 & 0.991195 & $\begin{array}{l}{[1145.017,} \\
1146.0223]\end{array}$ & 0.016142 & 59.628 & 0.99998 & -0.00517 & 3.06 \\
\hline$c_{p}$ & 3911.158 & 917.8 & 0.99181 & $\begin{array}{l}\text { [3917.778, } \\
3917.884]\end{array}$ & 0.022299 & 64.508 & 0.99992 & -0.063 & 3.08 \\
\hline$U$ & 9931.5342 & 10171 & 0.97344 & $\begin{array}{l}{\left[\begin{array}{l}10170.319, \\
10171.324]\end{array}\right.} \\
\end{array}$ & 0.014898 & 65.456 & 0.99998 & -0.0125 & 3.09 \\
\hline$A$ & 0.0148386 & 1.5279 & 0.71386 & \begin{tabular}{|l|}
1.52593 \\
$1.52989]$ \\
\end{tabular} & 0.061221 & 452.28 & 0.39106 & 0.324 & 3.08 \\
\hline$F_{H}$ & $\begin{array}{l}46.4 \\
\times 10^{-6} \\
\end{array}$ & 0.005055 & 0.002363 & $\begin{array}{l}{[0.005048,} \\
0.005062]\end{array}$ & 0.000203 & 449.29 & 0.39138 & 0.326 & 3.08 \\
\hline$V_{H}$ & $\begin{array}{l}50.4 \\
\times 10^{-6} \\
\end{array}$ & 0.005124 & 0.002436 & $\begin{array}{l}{[0.0051169,} \\
0.0051304]\end{array}$ & 0.000206 & 392.98 & 0.38563 & 0.389 & 3.17 \\
\hline$\rho_{H}$ & 977.79746 & 971.37 & 0.96991 & $\begin{array}{l}\text { [971.363, } \\
971.389]\end{array}$ & 0.022239 & 69.989 & 0.99957 & 0.0489 & 3.13 \\
\hline$c_{p H}$ & 4174.3783 & 4043.1 & 0.97664 & \begin{tabular}{|l}
4043.074, \\
$4043.179]$
\end{tabular} & 0.015468 & 65.86 & 0.99996 & 0.0459 & 3.12 \\
\hline
\end{tabular}

\subsection{Partial rank correlation coefficients for the model parameters}

Sensitivity analysis interpreted via partial rank correlation coefficients (PRCCs), is a technique that quantifies the uncertainty in the responsive model from the uncertainties of input initial conditions and parameters. Thus, to study the influence of uncertainties on the proposed model (1), we performed the global sensitivity analysis of the model which is a necessary and important tool to quantify the effects of uncertainties of the parameters on the responsive variables of the model. Based on the nature of the model, various methods for the sensitivity analysis have been proposed. For example, for non-linear models, methods that are based on decomposition of the output variance of the model like sobol method are used to quantify the model uncertainty but this is applicable for nonmonotonic models. For the case of nonlinear and monotonic models, spearman rank 


\section{J.P. Muhirwa, I.S. Mbalawata and V.G. Masanja}

correlation coefficients, standardized rank regression coefficients and the PRCCs are preferably to be used, but the PRCCs are the most accurate and adequate to measure and quantify the uncertainty in the outputs of the model [32]. The PRCCs vary in the range of \pm 1 with significant correlation for the values approaching -1 or +1 and low correlation for the values that are far from -1 or +1 . In this paper, the model (1) is nonlinear and monotonic, so the Latin Hypercube Sampling ((LHS) method with the PRCCs are implemented to qualitatively and quantitatively performing the sensitivity analysis. The obtained results are presented in Figure 10 and Table 3.

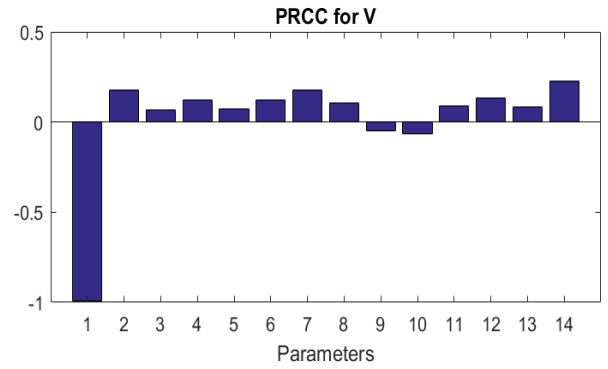

PRCC for T

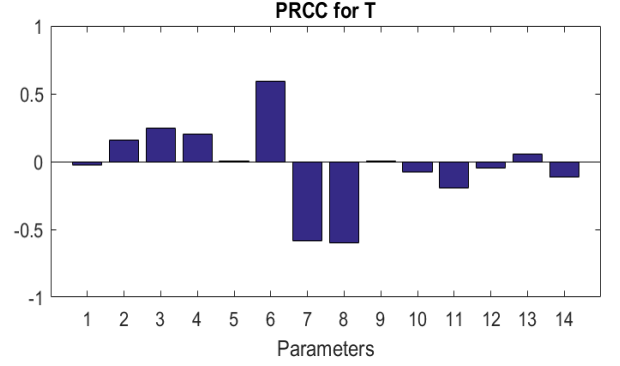

PRCC for C

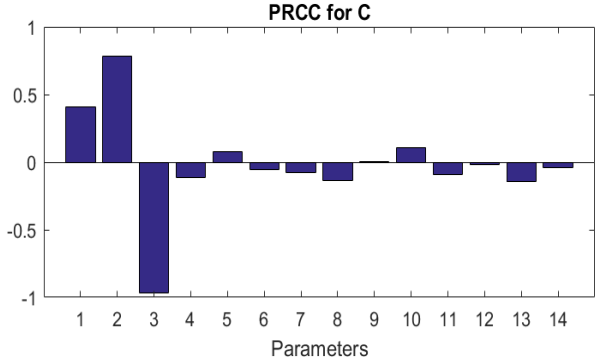

PRCC for $\mathrm{T}_{\mathrm{H}}$

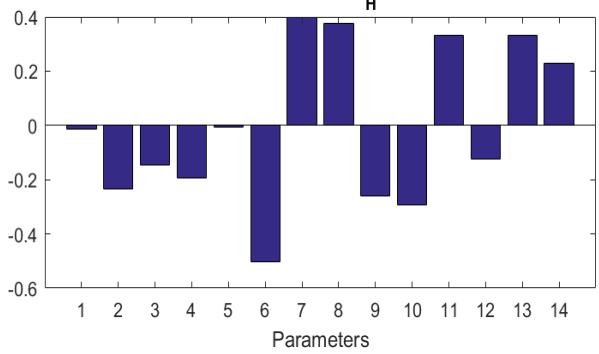

Figure 9: PRCCs plots

Figure 9, subplots 1-4 displays the PRCCs for the volume, the concentration, the temperature and the heater temperature respectively. This figure shows that the first parameter $\left(F_{\text {out }}\right)$ is strongly and negatively correlated with the volume means that the increase in the values of $F_{\text {out }}$ decreases the values of the volume in the reacting tank. Likewise, the second parameter $(F)$ is positively correlated with the concentration whilst the third one $\left(k_{0}\right)$ is significantly and negatively correlated with the concentration, and consequently, the increase in the values of $F$ will increase the values of the concentration in the model and the increase of $k_{0}$ will decrease the concentration of the model as can be seen from subplot 2. From subplot 3, we can explore that the increase of sixth parameter $\left(H^{*}\right)$ values will increase the temperature of the tank whereas the increase of the seventh $(\rho)$ and the eighth $\left(c_{p}\right)$ parameters values will automatically decrease the temperature values in the presented model. Subplot 4 shows that only sixth parameter is negatively correlated with the heater temperature which explains that the increase in the enthalpy values will directly inhibit the increase of the heater temperature values. As a result, $F_{\text {out }}, F, k_{0}, H^{*}, \rho$ and $c_{p}$ parameters are identified to be very 
Sensitivity and Uncertainty Analysis of Variable-Volume Deterministic Model for Endothermic Continuously Stirred Tank Reactor

sensitive to the model and hence much attention has to be quantitatively and qualitatively accorded to them.

Table 3: PRCCs values for each responsive model variable.

\begin{tabular}{|ccccc|}
\hline Parameters & & & Variables & \\
& $V$ & $C$ & $T$ & $T_{H}$ \\
$F_{\text {out }}$ & $-0.9890^{(*)}$ & 0.4067 & -0.0263 & -0.0126 \\
$F$ & 0.1793 & $0.7850^{(*)}$ & 0.1599 & -0.2350 \\
$k_{0}$ & 0.0645 & $-0.9678^{(*)}$ & 0.2452 & -0.1453 \\
$E$ & 0.1209 & -0.1126 & 0.1999 & -0.1934 \\
$T_{\text {mean }}$ & 0.0730 & 0.0739 & 0.0048 & -0.0053 \\
$H^{*}$ & 0.1246 & -0.0532 & $0.5897^{(*)}$ & $-0.5029^{(*)}$ \\
$\rho$ & 0.1777 & -0.0739 & $-0.5858^{(*)}$ & 0.3995 \\
$c_{p}$ & 0.1076 & -0.1335 & $-0.6017^{(*)}$ & 0.3773 \\
$U$ & -0.0473 & 0.0001 & 0.0060 & -0.2588 \\
$A$ & -0.0664 & 0.1083 & -0.0800 & -0.2929 \\
$F_{H}$ & 0.0918 & 0.0950 & -0.1923 & 0.3310 \\
$V_{H}$ & 0.1325 & -0.0212 & -0.0447 & -0.1234 \\
$\rho_{H}$ & 0.0846 & -0.1467 & 0.0563 & 0.3318 \\
$c_{p H}$ & 0.2289 & -0.0405 & -0.1175 & 0.2282 \\
\hline
\end{tabular}

Note: value ${ }^{(*)}$ means significant PRCCs values for the model variables.

\section{Conclusion}

In this paper, we formulated and numerically solved and analysed the variable-volume deterministic model for the endothermic continuously stirred tank reactor by using the least squares and the MCMC methods. The MCMC results have been graphically and statistically analysed to not only study the convergence of the method and the robustness of the model but to also examine the reliability of the model by identifying its physical parameters. We also performed the global sensitivity analysis to quantify the effect of uncertainty in the model from the uncertainty of estimated parameters by using LHS method, in order to obtain the PRCCs and their sensitivity significances for each variable of the model. Six parameters among fourteen identified parameters were shown to be correlated with the model variables and are very sensitive to the model responses (output). In the meantime, the numerical results have revealed that the model can be very beneficial to qualitatively, quantitatively and experimentally describe the dynamics of the variable-volume CSTR systems which require the heating process.

Acknowledgements. The authors express gratitude to anonymous referees for their critical supports as well as the German Academic Exchange Service (DAAD) financial support received by the first author to pursue his $\mathrm{PhD}$ studies at NM-AIST. In addition, the authors acknowledge publication fee received from African Institute for Mathematical Sciences (AIMS)-Secretariat in Kigali, Rwanda. 
J.P. Muhirwa, I.S. Mbalawata and V.G. Masanja

\section{REFERENCES}

1. P.Skupin, P.Laszczyk and M.Metzger, Bifurcation analysis of a hybrid continuous stirred tank reactor with imperfect mixing in the cooling jacket, IFAC-PapersOnLine, 52 (2019) 334-339.

2. N.G.Kanse, P.M.Dhanke and T.Abhijit, Modeling and simulation study of the CSTR for complex reaction by using polymath, Research Journal of Chemical Sciences, 2 (2012) 79-85.

3. N.Kumar and N.Khanduja, Mathematical modelling and simulation of CSTR using MIT rule, 2012 IEEE 5th India International Conference on Power Electronics (IICPE), 2012, 1-5.

4. F.A.Neba, H.M.Tornyeviadzi, S.W.Østerhus and R.Seidu, Self-optimizing attainable regions of the anaerobic treatment process: Modeling performance targets under kinetic uncertainty, Water Research, 171 (2020) 115377.

5. E.A.Buehler, J.A.Paulson, and A.Mesbah, Lyapunov-based stochastic nonlinear model predicitive control: Shaping the state probability distribution functions, in 2016 American Control Conference (ACC), 2016.

6. E.H.Karimi and K.B.McAuley, A bayesian method for estimating parameters in stochastic differential, IFAC-papersOnline, 48 (2015) 147-152.

7. E.Vlahakis and G.Halikias, Temperature and concentration control of exothermic chemical processes in continuous stirred tank reactors, Transactions of the Institute of Measurements and Control, 41 (2019) 4274-4284.

8. G.L.Foutch and A.H.Johannes, Reactors in process engineering, Encyclopedia of Physical Science and Technology, 2003, 00654-2.

9. Y.Lu, Z.Fang, and C.Gao, Stabilization of (state, input)-distributed cstrs through the port-hamilitonian systems approach, arXiv preprint arXiv: 1707.01560, 2017

10. B.G.Osorio, H.B.Castro, and J.D.S.Torres, State and unknown input estimation in a cstr using higher-order sliding mode observer, in IX Latin American Robotics Symposium and IEEE Colombian Conference on Automatic Control, 2011 IEEE, 2011.

11. J.Vojtesek and P.Dostal, Simulation of adaptive control of continuous stirred tank reactor, International Journal of Simulation Modelling, 8 (2009).

12. M.L.Johnson and L.M.Faunt, parameter estimation by least-squares methods, Methods in Enzymology, 210 (1992) 1-37.

13. G.Deepa Rani, U.M.Alhaz and U.M.Wali, An approximate technique for solving second order strongly nonlinear differential systems with high order nonlinearity in presence of small damping, Journal of Mathematics and Informatics, 5 (2016) 1-9.

14. L.Jin-Jun, P.Yu and C.Xi-Ming, Testing the dark energy model with strong gravitational lens via a markov chain Monte Carlo approach, Journal of Mathethatics and Informatics, 7 (2017) 79-86.

15. Y.Zhang and C.Wang, Approximation of Markov integrated semigroup induced by dual markov branching process and its application to signal processing, Journal of Mathematics and Informatics, 2 (2014) 12-16.

16. F.Remo, L.S.Luboobi, I.S.Mabalawata and B.K.Nannyonga, A mathematical model for the dynamics and mcmc analysis of tomato bacterial wilt disease, International Journal of Biomathematics , 11 (2018) 1850001. 
Sensitivity and Uncertainty Analysis of Variable-Volume Deterministic Model for Endothermic Continuously Stirred Tank Reactor

17. H.Haario, E.Saksman, J.Tamminen, et al., An adaptive metropolis algorithm, Bernoulli, 7 (2001) 223-242.

18. H.Haario, E.Saksman and J.Tamminen, Adaptive proposal distribution for random walk metropolis algorithm, Computational Statistics, 14 (1999) 375-396.

19. I.S.Mbalawata, S.Sarkka, M.Vihola and H.Haario, Adaptive methropolis algorithm using variational bayesian adaptive kalman filter, Computational Statistics \& Data Analysis, 83 (2015) 101-115.

20. J.P.Muhirwa and D.Ndanguza, Effect of random noise, quasi random noise and systematic random noise on unknown continuous stirred tank reactor (cstr), Applied Mathematical Sciences, 11 (2017) 3051-3071.

21. J.Vojtesek and P.Dostal, Simulation analysis of continuous stirred tank reactor, IEEE Trans. Automat. Control, 24 (2008) 27-31.

22. M.A.Aboelela and R.H.M.Hennas, Development of a fractional order pid controller using adaptive weighted pso and genetic algorithms with applications, in: Fractional Order Systems, Elsevier, (2018) 511-551.

23. S.Brooks, Markov chain monte carlo method and its application, Journal of the Royal Statistical Society: Series D, 47 (1998) 69-100.

24. V.Roy, Convergence diagnostics for markov chain monte carlo, Annual Review of Statistics and its Application, 7 (2020) 387-412.

25. S.Sharma, Markov chain monte carlo methods for bayesian data analysis in astronomy, Annual Review of Astronomy and Astrophysics, 55 (2017) 213-259.

26. S.Sinharay, Assessing convergence of the Markov chain Monte Carlo algorithms: A review, ETS Research Report Series, 2003 (2003) i-52.

27. M.K.Cowles and B.P.Carlin, Markov chain Monte Carlo convergence diagnostics: A comparative review, Journal of the American Statistical Association, 91 (1996) 883904.

28. A.Gelman and D.B.Rubin, Inference from iterative simulation using multiple sequences, Statistical Science, 45 (1992) 457-511. 